
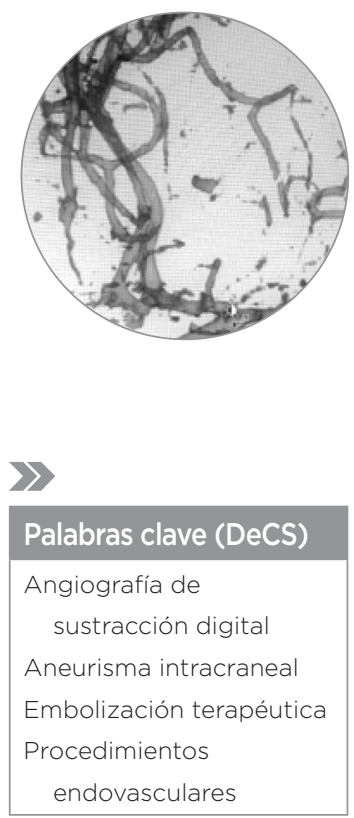

\section{Key words (MeSH)}

Digital subtraction

angiography

Intracranial aneurysm

Embolization, therapeutic

Endovascular procedures

\title{
Utilidad de la angiografía por sustracción digital 3D en el abordaje endovascular de aneurismas cerebrales
}

\author{
Usefulness of 3D Digital Subtraction Angiography in the Endovascular \\ Approach of Cerebral Aneurysms
}

\author{
Nelson Oswaldo Lobelo García' \\ Alejandra Navarrete Sánchez ${ }^{2}$ \\ Mauricio Enrique Moreno Mejía ${ }^{3}$ \\ Cristian Camilo Páez ${ }^{4}$ \\ Cihara Valessa Avendaño Padilla ${ }^{5}$
}

\begin{abstract}
Resumen
Introducción: En el manejo de los aneurismas intracraneales la tendencia ha sido realizar procedimientos endovasculares mínimamente invasivos. Nuevas herramientas en imágenes, como la angiografía por sustracción digital en 3D (ASD 3D), sumadas a la experiencia de los radiólogos neurointervencionistas, han llevado a una mayor definición y precisión en el estudio del aneurisma intracraneal. Objetivo: Describir la utilidad de la técnica de angiografía por sustracción digital tridimensional para el abordaje pre y postembolización de los aneurismas intracraneales. Metodología: Estudio de corte transversal entre enero de 2016 y abril de 2017 en pacientes diagnosticados con aneurisma de arterias cerebrales, en el Hospital Infantil Universitario San José, en Bogotá, Colombia. Resultados: Se incluyeron 32 pacientes, de los cuales 71,8 \% $(n=23)$ fueron mujeres. Entre los factores de riesgo para ruptura del aneurisma, el más frecuente fue edad mayor a 40 años (81,8 \%). La localización más usual fue en la arteria cerebral media (ACM) derecha (30,3 \%). Todos los casos correspondieron a aneurismas saculares. En el control angiográfico postembolización inmediato se evidenció que 16 casos (48,5 \%) presentaron saco residual. Conclusiones: La realización de proyecciones multiplanares con reconstrucción angiográfica 3D brinda información adicional para una mejor caracterización del aneurisma y evaluación de las estructuras anatómicas adyacentes, por lo que es de gran utilidad para planear el procedimiento y para el seguimiento.
\end{abstract}

\section{Summary}

Introduction: The trend in management of intracranial aneurysms has shifted during the last decades to minimally invasive endovascular procedures. The usefulness of new imaging tools such as digital subtraction angiography in 3D (3D DSA), added to the experience of neurointerventional radiologists, have led to greater definition and accuracy in the study of intracranial aneurysms. Objective: To describe the usefulness of three-dimensional digital subtraction angiography for pre and post embolization approach of intracranial aneurysms. Methodology: A cross-sectional study between January 2016 and April 2017 in patients diagnosed with arterial cerebral aneurysms at the Hospital Infantil Universitario San José in Bogota, Colombia. Results: 32 patients were included, 71.8\% $(n=23)$ were women. Among the risk factors for aneurysm rupture, the most frequent was age above 40 years (81.8\%). The most frequent location was in the Right Middle Cerebral Artery (MCA) (30.3\%). All cases corresponded to saccular aneurysms. In the immediate post-embolization angiographic control it was evidence that 16 cases (48.5\%) presented residual sac. Conclusions: The realization of multiplanar projections with 3D angiographic reconstruction allows for a better characterization of the aneurysm and evaluation of the adjacent anatomical structures, being very useful for the planning of the procedure and in the follow-up.

\section{Introducción}

En las últimas décadas, la tendencia en el manejo de los aneurismas intracraneales está basada en procedimientos endovasculares mínimamente invasivos, lo cual demuestra que el tratamiento intravascular comparado con el tratamiento quirúrgico reduce el riesgo de muerte o discapacidad a largo plazo (1).
Los factores implicados en la realización de un adecuado procedimiento dependen cada vez más de la utilidad de nuevas herramientas en imágenes, como la angiografía por sustracción digital en 3D (ASD 3D) y de la experiencia de los radiólogos neurointervencionistas, que facilita una mayor definición y precisión en la caracterización del aneurisma intracraneal, el estado fisiológico de los vasos comunicantes (vasoespasmo),
Institución: Diaimagen, Departamento de Imágenes diagnósticas. Hospital Infantil Universitario de San José. 
así como de las estructuras anatómicas. Lo anterior, disminuye el tiempo de los procedimientos y a su vez, la frecuencia de complicaciones (2-4).

La ASD con proyecciones en 2D se empleó como la modalidad de elección para el estudio de las estructuras vasculares intracraneales por varios años, con imágenes en los planos anteroposterior, lateral y oblicuos. La ASD 3D es una evolución tecnológica de la ASD 2D que permite visualizar los vasos intracraneales desde cualquier proyección posible. Para la ASD 3D se requiere de un angiógrafo de detector plano con un brazo en $\mathrm{C}$ rotativo. Se realizan dos adquisiciones de imágenes rotacionales, la primera para obtener una máscara de substracción y la segunda adquiere las imágenes durante la inyección del medio de contraste. En la segunda adquisición, el arco rota 180 grados en aproximadamente 4-8 segundos, a la vez que se administran $13 \mathrm{ml}$ de medio de contraste a través de un inyector a una tasa de $2,5 \mathrm{~mL} / \mathrm{s}$. Las subsecuentes reconstrucciones con un software especializado permiten la supresión de vasos innecesarios, rotación de la imagen y magnificación, entre otras aplicaciones (2).

El propósito de este artículo es ilustrar con casos clínicos la utilidad de la angiografía por sustracción digital en 3D en el abordaje endovascular de aneurismas cerebrales y comparar los resultados encontrados antes de la intervención y en el postoperatorio inmediatos con lo publicado en la literatura.

\section{Metodología}

Se realizó un estudio de corte transversal entre enero de 2016 y abril de 2017 con pacientes diagnosticados con aneurisma de arterias cerebrales. Se incluyeron 32 pacientes a quienes se les realizaron angiografías por sustracción digital con reconstrucción 3D; se hizo diagnóstico de 33 aneurismas; todos ellos fueron tratados mediante embolización con espirales (coils). Como variables independientes se tuvieron en cuenta el sexo, la edad, los factores de riesgo para formación de aneurismas cerebrales en cada paciente; la localización, tamaño y forma del aneurisma, las características del vaso aferente y los vasos que se originan en el saco del aneurisma. También se registraron: la duración del procedimiento, los resultados postratamiento y las características del vaso aferente en la angiografía 3D de control postratamiento inmediato.

Los datos se obtuvieron de la estación de trabajo de un angiógrafo TOSHIBA modelo INFX-8000V/W6, Serie W6C12Y2019, utilizando la aplicación VÍTREA FX (Versión 6.3). Los demográficos y clínicos de las historias clínicas de cada paciente.

Para la realización de la angiografía se siguió el protocolo institucional en cada uno de los pacientes y posteriormente se practicó reconstrucción 3D con el software mencionado.

Se hizo un análisis univariado de las variables incluidas para establecer frecuencias absolutas y relativas mediante el paquete estadístico SPSS Statistics V24.0. No se tuvieron datos ausentes.

Se llevó a cabo una búsqueda bibliográfica en las bases de datos PubMed, OVID y ClinicalKey, con las palabras clave angiografía de sustracción digital, aneurismas intracraneales, embolización terapéutica y procedimientos endovasculares, para comparar los resultados obtenidos con lo descrito en la literatura.

Se contó con la aprobación del comité de ética médica del hospital y se cumplió la normatividad vigente en Colombia.

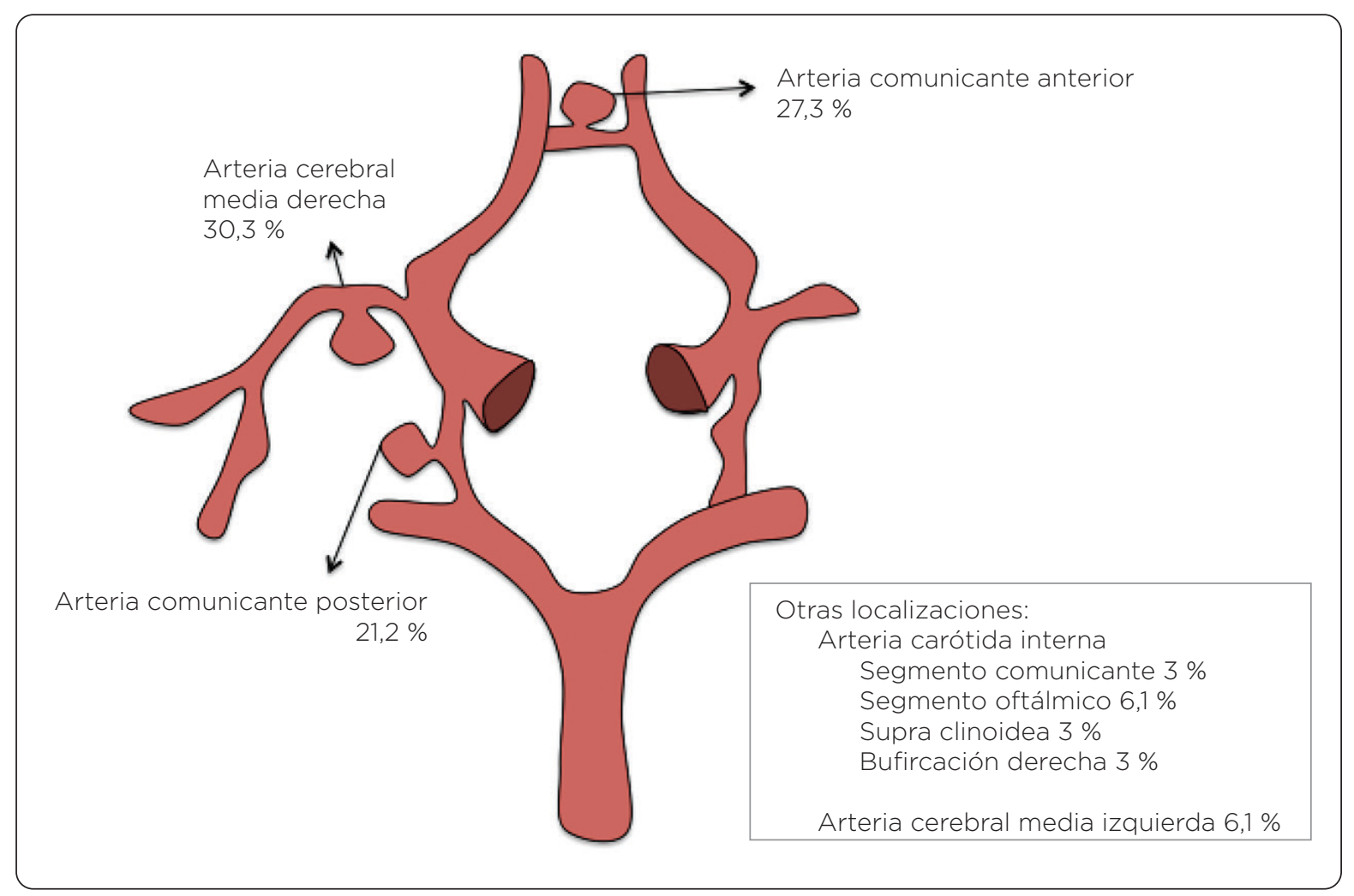

Figura 1. Representación gráfica de la localización más frecuente de los aneurismas en la población estudiada. 


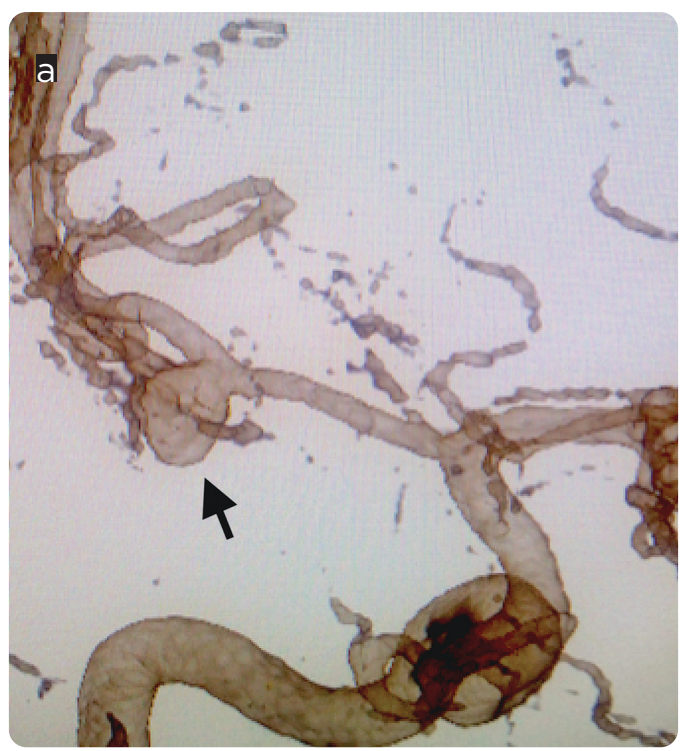

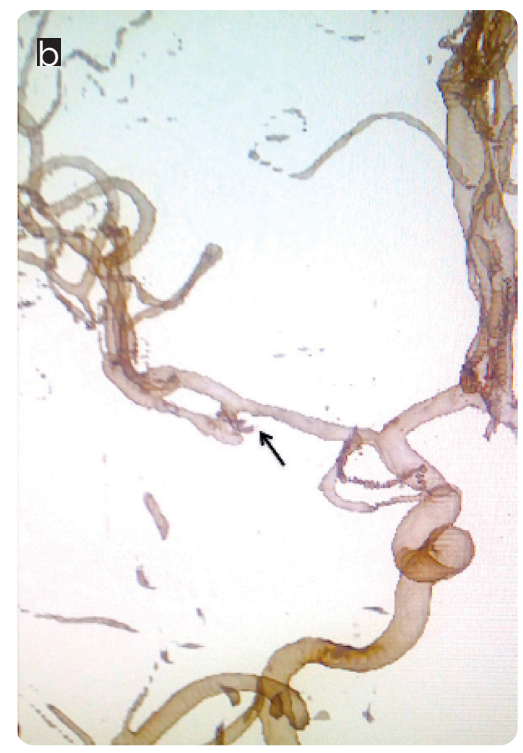

Figura 2. Aneurisma sacular de la arteria cerebral media derecha. a). Reconstrucción 3D preembolización (flecha). b) Reconstrucción 3D postembolización. Obsérvese la porción de cuello del saco aneurismático remanente en la arteria cerebral media derecha (flecha).
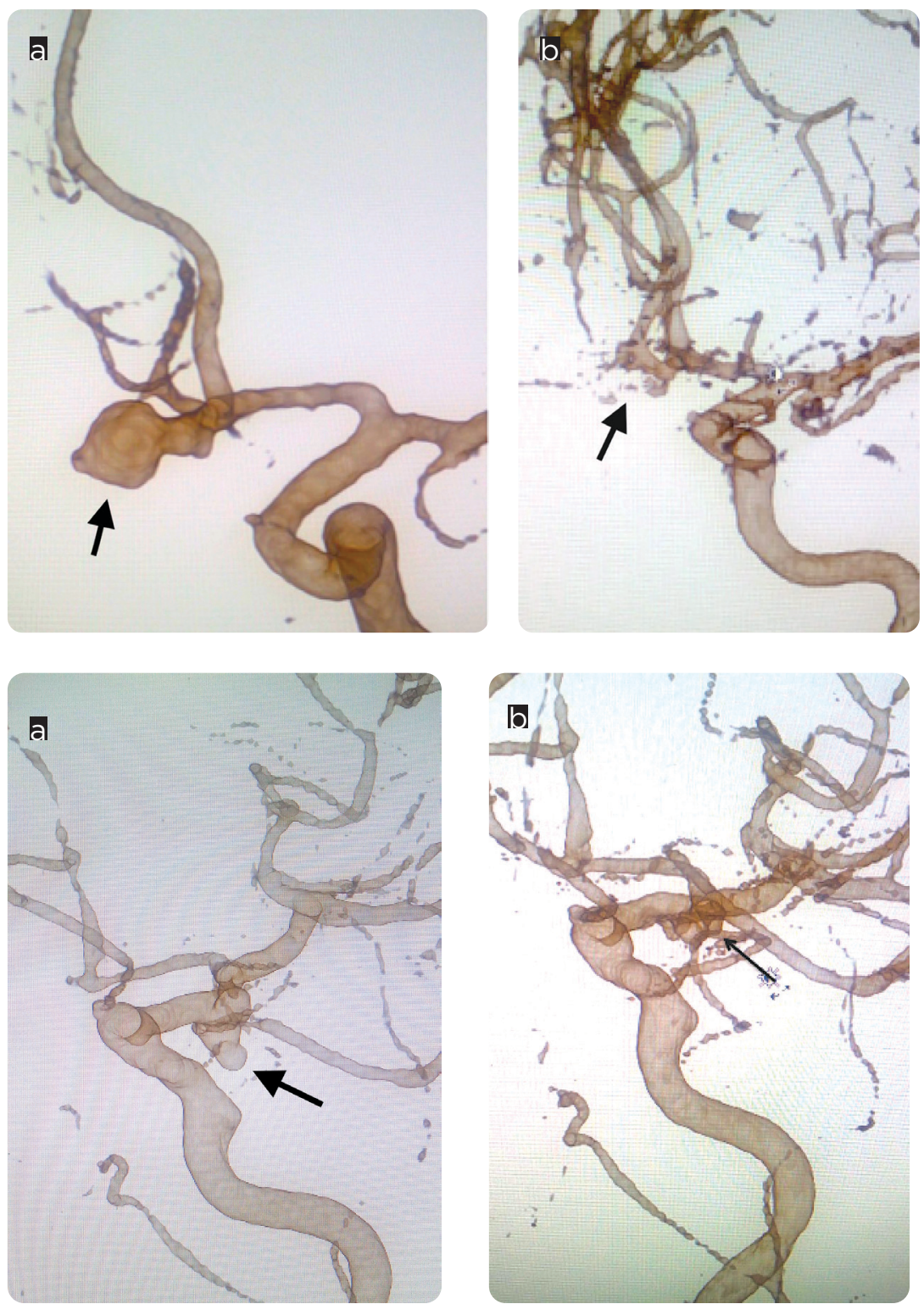

Figura 3. Aneurisma sacular de la arteria comunicante anterior. a) Reconstrucción 3D preembolización (flecha). b). Reconstrucción 3D postembolización. Obsérvese la oclusión completa del saco aneurismático (flecha).

Figura 4. Aneurisma sacular de la arteria comunicante posterior. a) Reconstrucción 3D preembolización (flecha) b) Reconstrucción 3D postembolización. Obsérvese la porción de saco aneurismático remanente en la arteria comunicante posterior (flecha). 


\section{Resultados}

De los 32 pacientes incluidos en el estudio, $71,8 \%(n=23)$ fueron mujeres y 29,2 \% hombres $(n=9)$. La edad mínima fue de 3 años y la máxima de 90 años, con una media de 55,5 años (Desviación estándar [DE] de 17,9 años). Entre los factores de riesgo para ruptura del aneurisma se encontró edad mayor a 40 años en 27 pacientes $(81,8 \%)$, 17 pacientes eran hipertensos $(53,1 \%)$ y 5 eran fumadores $(15,6 \%)$. En la tabla 1 se muestran las características de la población estudiada. Las localizaciones más frecuentes fueron: arteria cerebral media (ACM) derecha con 10 casos $(30,3 \%)$, arteria comunicante anterior (ACoA) con nueve casos $(27,3 \%)$ y arteria comunicante posterior (ACoP) con siete casos (21,2\%) (figura 1). El tamaño de los aneurismas osciló entre $3,6 \mathrm{~mm}$ y $26 \mathrm{~mm}$, con promedio de $8,44 \mathrm{~mm}$; hubo mayor frecuencia de aneurismas pequeños (menores a $10 \mathrm{~mm}$ ), que correspondieron al $93,9 \%(n=31)$. En cuanto a las características del vaso aferente, se documentó que en la mayoría de los casos era normal (60,6 \%). En 21 aneurismas $63,6 \%$ de los casos hubo vasos que se originaban en el saco del aneurisma. Todos los casos correspondieron a aneurismas saculares (figura 2). En el control angiográfico postembolización se evidenció que 16 casos $(48,5 \%)$ presentaron saco residual (figuras 3 y 4 ) y que las características del vaso aferente eran normales en la mayoría de estos, 23 casos (69,7 \%) (figura 5). La duración del procedimiento estuvo en el rango de 14 a 106 minutos con promedio de 39 minutos (tabla 2).

\section{Tabla 1. Características de la población estudiada}

\begin{tabular}{|l|c|c|}
\hline \multicolumn{1}{|c|}{ Variable } & $n$ & $\begin{array}{c}\text { Porcentaje } \\
\text { (\%) }\end{array}$ \\
\hline Sexo & 23 & 69,7 \\
\hline Mujer & 9 & 27,3 \\
\hline Hombre & & \\
\hline Edad & 5 & 18,2 \\
\hline Menores de 40 años & 27 & 81,8 \\
\hline Mayores de 40 años & & \\
\hline Factores de riesgo de ruptura de aneurisma & 17 & 53,1 \\
\hline Hipertensión arterial & 5 & 15,6 \\
\hline Fumadores & 1 & 3,1 \\
\hline Malformación arteriovenosa asociada & 4 & 12,5 \\
\hline Ninguno & & \\
\hline
\end{tabular}

\section{Discusión}

Los aneurismas intracraneales son dilataciones externas anormales en la pared de los vasos sanguíneos intracraneales; la variante sacular es la más común. Están localizados con frecuencia en la circulación anterior $(90 \%)$ y afectan comúnmente mujeres en la $5 .^{\text {a }}$ década de la vida (5-7), características todas frecuentes en la población de este estudio. Aún se desconoce la etiología de los aneurismas saculares (8); sin embargo, se han estudiado factores de riesgo predisponentes para el desarrollo y ruptura de estos, como la hipertensión arterial sistémica y el tabaquismo, presentes en el 53,1 \% y 15,6 \% de nuestros pacientes, respectivamente. Otro factor de riesgo per se es la edad mayor a 40 años, que correspondió al $81,8 \%$ de la población. En esta investigación con más de un año de seguimiento no hubo casos de ruptura del aneurisma, probablemente influenciado por otros factores como: la mayoría de los pacientes presentaron aneurismas pequeños y se obtuvo oclusión completa o solo cuello residual en más de la mitad de los casos.

Algunos autores han considerado la ASD 3D como la imagen diagnóstica de referencia para la evaluación de la circulación intracraneal en general y la planeación del tratamiento de los aneurismas cerebrales, y se han encontrado complicaciones tan bajas como del 0,3\% en manos expertas (9).

Estudios previamente publicados han demostrado la capacidad superior de la ASD 3D al compararla con la 2D ASD y la angiografía rotacional, evidenciando la posibilidad de adquisición de imágenes en el eje cráneo-caudal en alta resolución, la eliminación de estructuras fabricadas de material metálico prótesis y espirales (stents, coils) o estructuras superpuestas y la realización de procesos de simulación. Además, es una técnica hemodinámica intracraneal, que logra en tiempo real valorar el estado fisiológico de los vasos colaterales, del vaso aferente del aneurisma y los vasos dentro del saco aneurismático $(10,11)$.

En nuestra investigación, en cuanto al tamaño del aneurisma con la técnica 3D ASD se pudieron detectar aneurismas pequeños desde $3,6 \mathrm{~mm}$. Investigaciones previas han documentado que la angiografía $3 \mathrm{D}$ puede detectar aneurismas hasta de $0,5 \mathrm{~mm}$.

A la angiografía 2D se le ha atribuido una sensibilidad y especificidad mayor al $90 \%$ para detectar aneurismas que miden $3 \mathrm{~mm}$; sin embargo, tiene marcadas limitaciones para visualizar aneurismas menores a $2 \mathrm{~mm}(12,13)$.

En los 33 aneurismas de nuestro estudio se pudo clasificar adecuadamente su forma como sacular y se obtuvieron imágenes de una calidad suficiente para planear el tratamiento endovascular. La literatura describe que la capacidad de la angiografía $2 \mathrm{D}$ para mostrar con precisión la forma del aneurisma tiene un menor rendimiento en comparación con las imágenes de la 3D ASD (3).

Respecto a la definición de las características del vaso aferente y la presencia de vasos originados en el saco del aneurisma, esta técnica logra eliminar la superposición de estructuras vasculares, gracias al posproceso en la consola de trabajo y a la vista en 360 grados del aneurisma. De esta manera se pudo detectar que en el $18,2 \%$ de los casos el vaso aferente era displásico y que el 63,6 \% de los casos existían vasos en el saco aneurismático. Aunque no se describió específicamente el rol de la 3D ASD para evitar la sobreposición de estructuras vasculares en la presente investigación, se han publicado varios estudios que apoyan la superioridad de esta característica la 3D ASD sobre la 2D al caracterizar estos hallazgos (11).

En algunos estudios se recomienda la ASD 3D como el estudio estándar para seguimiento angiográfico de pacientes con aneurismas embolizados con espirales (coils), para incrementar la tasa de detección de aneurismas remanentes e identificar la estenosis de los vasos aferentes $(6,8,9,12)$. En nuestro estudio se identificó el saco aneurismático remanente (la mayoría de pequeño tamaño), como el principal resultado postratamiento inmediato, en un 48,5\%. 

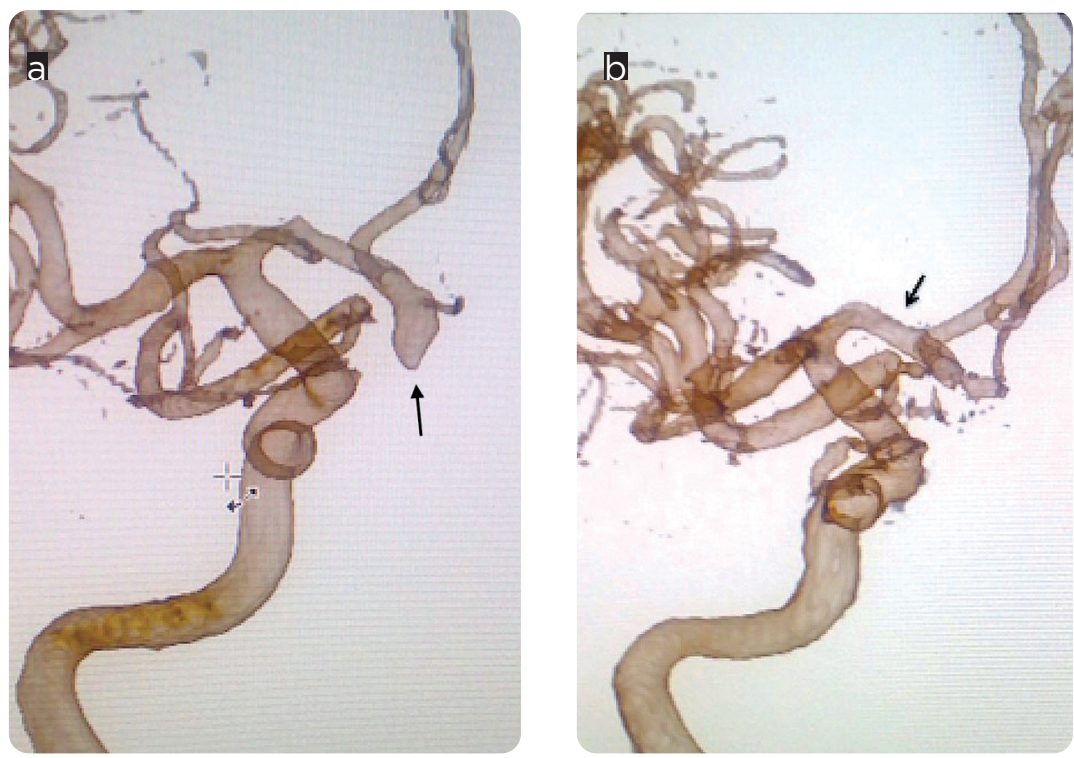

Figura 5. Aneurisma sacular de la arteria comunicante anterior. a) Reconstrucción 3D preembolización (flecha) b) Reconstrucción 3D postembolización. Obsérvese el estado normal del vaso aferente (flecha).

Tabla 2. Características evaluadas en la angiografía 3D del aneurisma y vaso aferente antes y después de la embolización

\begin{tabular}{|c|c|c|}
\hline Variable & $n$ & $\begin{array}{c}\text { Porcentaje } \\
(\%)\end{array}$ \\
\hline \multicolumn{3}{|c|}{ Tamaño } \\
\hline Pequeño ( $<10 \mathrm{~mm}$ ) & 31 & 94 \\
\hline Grande ( > $10 \mathrm{~mm}$ ) & 1 & 3 \\
\hline Gigante ( $>25 \mathrm{~mm}$ ) & 1 & 3 \\
\hline \multicolumn{3}{|c|}{ Características del vaso aferente } \\
\hline Normal & 20 & 60,6 \\
\hline Displásico & 6 & 18,2 \\
\hline Con vasoespasmo & 7 & 21,2 \\
\hline \multicolumn{3}{|c|}{ Vasos en el saco del aneurisma } \\
\hline Sí & 21 & 63,6 \\
\hline No & 12 & 36,4 \\
\hline \multicolumn{3}{|c|}{ Resultado postratamiento } \\
\hline Saco residual & 16 & 48,5 \\
\hline Cuello residual & 10 & 30,3 \\
\hline Oclusión completa & 7 & 21,2 \\
\hline \multicolumn{3}{|c|}{ Características del vaso aferente postembolización } \\
\hline Normal & 23 & 69,7 \\
\hline Con vasoespasmo & 6 & 18,2 \\
\hline Displásico & 3 & 9,1 \\
\hline Con estenosis & 1 & 3,0 \\
\hline
\end{tabular}

\section{Limitaciones del estudio y recomendaciones para estudios posteriores}

Nuestros hallazgos deben ser replicados con controles de seguimiento a mediano y largo plazo para determinar la recurrencia, disminución o estabilización del aneurisma, dado que, de acuerdo con el tamaño y la configuración del aneurisma, pueden ser necesarias reintervenciones posteriores para completar el tratamiento. Este seguimiento a mediano y largo plazo no fue posible, en parte debido a factores administrativos derivados del sistema de salud, ya que algunos pacientes fueron remitidos para su seguimiento y control en otras instituciones. Sin embargo, la gran mayoría de los pacientes fueron valorados por el médico tratante, con evidencia de cierre completo de los aneurismas. Adicionalmente, se considera que se debe aplicar una clasificación para determinar el porcentaje de saco remanente, con eso sería más fácil y objetiva la manera de comparar en los estudios de seguimiento las variaciones significativas que presenten los aneurismas.

\section{Conclusión}

Este estudio observacional muestra que la realización de proyecciones multiplanares con reconstrucción en 3D permiten una completa caracterización del aneurisma y evaluación de las estructuras anatómicas adyacentes, lo cual es de gran utilidad para planear el procedimiento y en el seguimiento postembolización, especialmente para determinar el grado de oclusión de los aneurismas tratados.

\section{Referencias}

1. Molyneux AJ, Kerr RSC, Yu L-M, et al. International subarachnoid aneurysm tria (ISAT) of neurosurgical clipping versus endovascular coiling in 2143 patients with ruptured intracranial aneurysms: a randomised comparison of effects on survival, dependency, seizures, rebleeding, subgroups, and aneurysm occlusion. Lancet (London, England). 2005;366(9488):809-17.

2. Cieściński J, Serafin Z, Strześniewski P, et al. DSA volumetric 3D reconstructions of intracranial aneurysms: A pictorial essay. Polish J Radiol. 2012;77(2):47-53.

3. Anxionnat R, Bracard S, Ducrocq X, et al. Intracranial aneurysms: Clinical Value of $3 \mathrm{D}$ digital subtraction angiography in the therapeutic decision and endovascular treatment. Radiology. 2001;218(3):799-808.

4. Tanoue S, Kiyosue H, Kenai H, et al. Three-dimensional reconstructed images after rotational angiography in the evaluation of intracranial aneurysms: Surgical correlation. Neurosurgery. 2000;47(4):866-71.

5. Robbins SL, Kumar V, Cotran RS. Robbins and Cotran pathologic basis of disease 8th ed. Philadelphia PA: Saunders/Elsevier; 2010.

6. Zhou B, Li M-H, Wang W, et al. Three-dimensional volume-rendering technique in the angiographic follow-up of intracranial aneurysms embolized with coils. J Neurosurg. 2010;112(3):674-80.

7. Hacein-Bey L, Provenzale JM. Current imaging assessment and treatment of intracranial aneurysms. Am J Roentgenol. 2011;196(1):32-44. 
8. Grobelny TJ. Brain aneurysms: Epidemiology, treatment options, and milestones of endovascular treatment evolution. Disease-a-Month. 2011;57(10):647-55.

9. Fifi JT, Meyers PM, Lavine SD, et al. Complications of modern diagnostic cerebral angiography in an Academic Medical Center. JVIR. 2009;20:442-7.

10. Bau Alegría J. Reconstrucción $3 \mathrm{D}$ angiográfica en el diagnóstico y el tratamiento de aneurismas cerebrales. Imagen Diagnóstica. 2010;1(2):51-5

11. Sugahara T, Korogi Y, Nakashima K, et al. Comparison of $2 \mathrm{D}$ and $3 \mathrm{D}$ digital subtraction angiography in evaluation of intracranial aneurysms. AJNR Am J Neuroradiol. 2002;23(9):1545-52.

12. Van Rooij WJ, Sprengers ME, de Gast AN, et al. 3D Rotational angiography: The new gold standard in the detection of additional intracranial aneurysms. Am J Neuroradiol. 2008;29(5):976-9.

13. Hochmuth A, Spetzger U, Schumacher M. Comparison of three-dimensional rotational angiography with digital subtraction angiography in the assessment of ruptured cerebral aneurysms. Am J Neuroradiol. 2002;23(7):1199-205.

\section{Correspondencia}

Mauricio Enrique Moreno Mejía

Hospital Infantil Universitario de San José

Carrera 52 \# 67A - 71

Bogotá, Colombia

mmorenomejia@hotmail.com

Recibido para evaluación: 1 de diciembre de 2019

Aceptado para publicación: 8 de marzo de 2020 